\title{
SDRG \\ Saudi Diabetes Research Group \\ Gamma Glutamyl Transferase and C- Reactive Protein in Type 2 Saudi Diabetic Patients in Relation to Management Modality and Components of Metabolic Syndrome
}

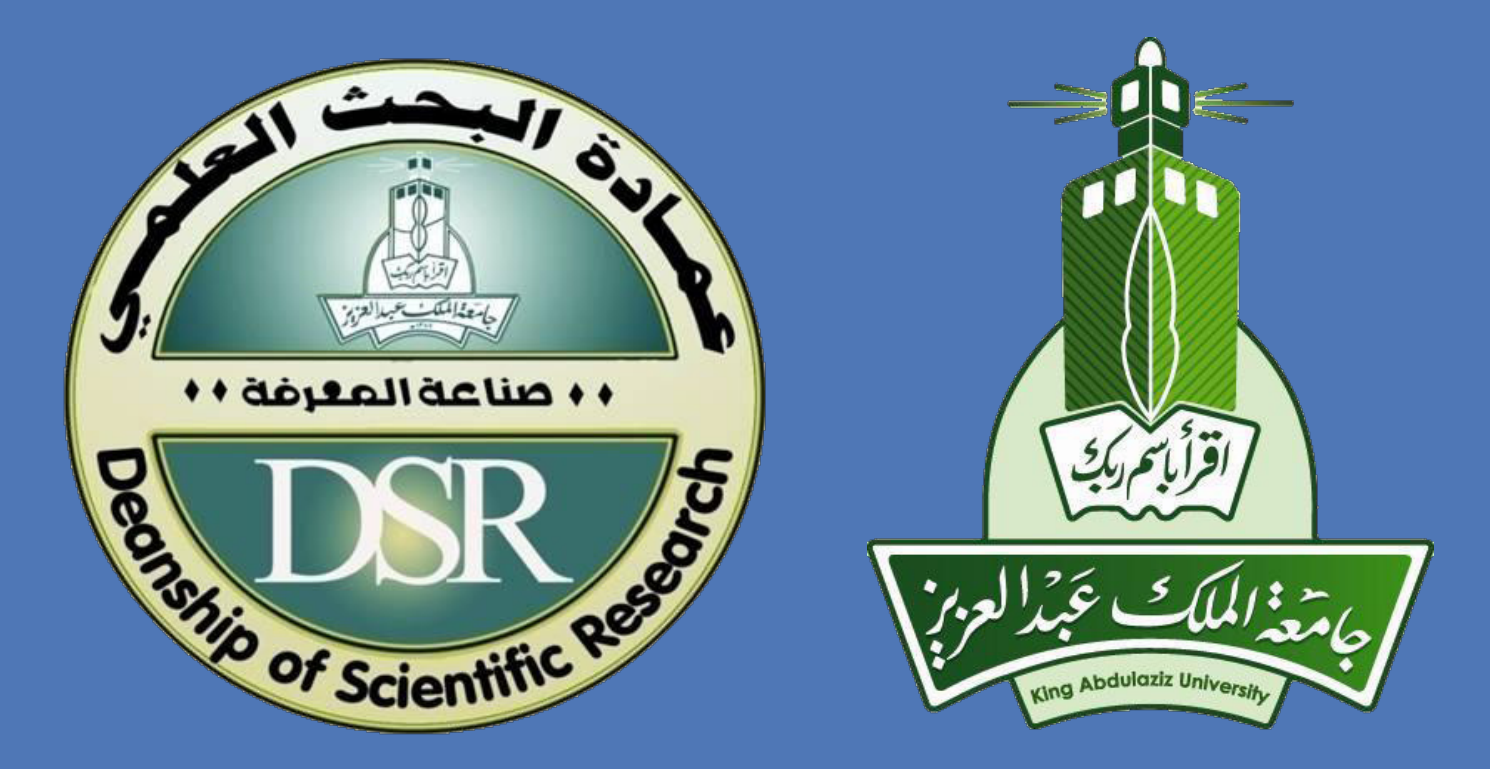

作

1 Dept. Clin. Biochem- Faculty of Medicine-
${ }^{2}$ Saudi Diabetes Research Group - KAU

3Dept. Internal Medicine - Faculty of Medicine- KAU
4King Fahad Armed forces hospital (KFAFH) in Jeddah

(KAU) SKing Abdullah International Medical Research Center, King Saud bin
Sciences, Pathology Dept., King Abdulaziz Medical City, Jeddah

University of Helsinki, Hjelt Institute, Department of Public Health, Helsinki, Finland

Sponsored by the Deanship of Scientific Research at KAU - Grant number T-094/429

\section{Abstract:}

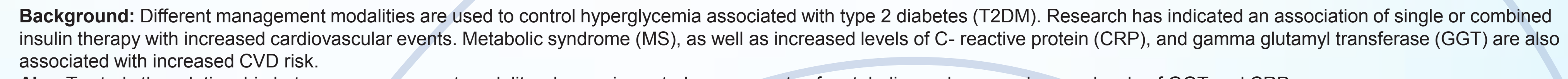

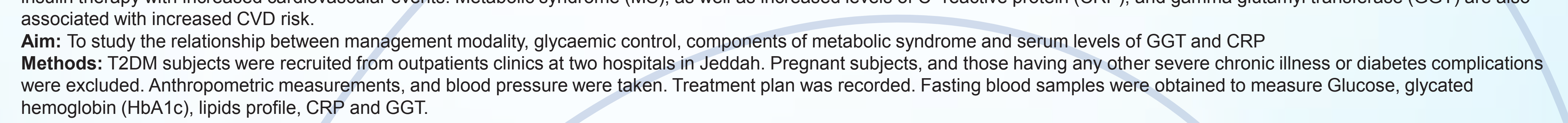

\section{Introduction:}

Type 2 diabetes mellitus (T2DM) is highly prevalent amongst the adult population of Saudi Arabia. Due to the increasing number of available choices to control the associated hyperglycemia, lack of Saudi guidelines, treating physicians have become increasingly uncertain of best management policy (1). Strict glycemic control over time has long been reported to decrease microvascular complications associated with the disease (2-4). In spite of the fact that insulin therapy is very effective in controlling all levels of hyperglycemia, as well as coexisting dyslipidemia (5), it was reported to increase frequency of severe hypoglycemic episode (6). In addition, use of dyslipidemia (5), it was reported to increase frequency of severe hypoglycemic episode (6). In addition, use of
insulin therapy was associated with increased risk of cardiovascular disease (CVD) directly ( 7 ), or indirectly due insulin therapy was associated with increased risk of cardiovascular disease (CVD) directly (7), or indirectly due
to weight gained following insulin therapy (8). Increased risk of CVD has also been associated with the presence to weight gained following insulin therapy (8). Increased risk of CVD has also been associated with the presenc
of metabolic syndrome $(9,10)$, as well as increased levels of C-reactive protein (CRP) $(11,12)$, and gamma glutamyl transferase (GGT) $(13,14)$. The aim of our study is to understand the relationship between management glutamyl transferase (GGT) $(13,14)$. The aim of our study is to understand the relationship between mant
modality, glycaemic control, components of metabolic syndrome and serum levels of GGT and CRP

\section{Methods:}

Patients diagnosed with type 2 diabetes mellitus, were randomly recruited from the diabetes out-patients clinics a King abdulaziz university hospital and King Fahad Armed forces hospital (KFAFH) in Jeddah, Saudi Arabia, during the course of one month ( 1 st of June to $2^{\text {nd }}$ of July, 2014). Ethical approval was obtained from the Committee on
the Ethics of Human Research at the "Faculty of Medicine- King Abdulaziz University", and the Committee on the the Ethics of Human Research at the "Faculty of Medicine- King Abdulaziz University", and the Committee
Ethics of Medical Research at KFAFH Willing patients were asked to sign an informed consent form to Ethics of Medical Research at KFAFH Willing patients were asked to sign an informed consent form to participate. Exclusion criteria included: pregnancy, having any other severe chronic illness or diabetes
complications (i.e. end stage renal disease, liver disease, recent myocardial infarction, etc). Blood pressure was measured following the recommendations of the American Heart Association Council (15), using a standard mercury sphygmomanometer with the cuff on the right upper arm. Two blood pressure readings; one minute apart; while the subject was seated for 10 minutes were taken, and the mean of the two readings was calculated. Anthropometric measurements were taken for all. Height was measured bare footed to the nearest $0.5 \mathrm{~cm}$ using stationary stadiometer. Weight was measured to the nearest $0.5 \mathrm{Kg}$ while wearing light street clothing using a calibrated scale. Both measurements were used to calculate body mass index (BMI). Waist measurements was taken at the level of the umbilicus, and hip measurement at the maximal protrusion of the gluteal muscles, both the nearest $0.5 \mathrm{~cm}$. BMI was used to classify patients as being normal $(18.5-<25)$, overweight $(25-<30)$, or was following to control his/her questionnaire comprising the demographic and management plan that the patient lifestyle modification (i.e diet and exercise) oral hypoglycemic agents (metofrmin sulfonylurea, was recor glucosidase inhibitor thiazolidinedione, or DPP4 inhibitor), insulin, non-insulin injectable drugs (GLP-1 agonist), or any combination of them. Fasting (HbA1c) lipids profile, high sensitivity CRP (hs- CRP) and GGT. Serum glucose and lipids (cholesterol triglycerides and high density lipoprotein (HDL-C) were assayed using automated enzymatic methods (Dimension Vista 1500T Intelligent Lab System from SIEMENS Company) at the biochemistry laboratory. Low density lipoprotein cholesterol (LDL-C) was calculated using the Friedewald equation (17). Glycated haemoglobin (HbA1c) analysis was performed on Dimension Vista 1500T Intelligent Lab System (SIEMENS Company)
$\leq 7 \%$ was considered controlled and $\mathrm{Hb}$ A1c of $>7 \%$ Was considered uncontrolled, in accordance to the American Diabetic Association Guidelines (18). Hypertension was defined as a systolic blood pressure $>140$ $\mathrm{Hg}$, and/ or diastolic blood pressure $>90 \mathrm{~mm} \mathrm{Hg}$ or current use of anti
consensus definition (20) was used to diagnose metabolic syndrome.

Statistical/Analysis: Data was entered, coded, and analyzed using SPSS version, 20. Descriptive statistics, such as mean \pm SD, were calculated for all estimated parameters. Comparison between two means was performed using unpaired Student t-test for normally distributed, and the Mann Whitney-U test for non-normally distributed parameters. Differences between more than two means were tested using One way ANOVA analysis. Chi square test was used for categorical variables. All $p$ values that were $<0.05$ were deemed statistically
significant.

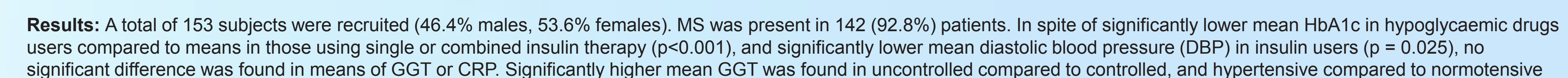

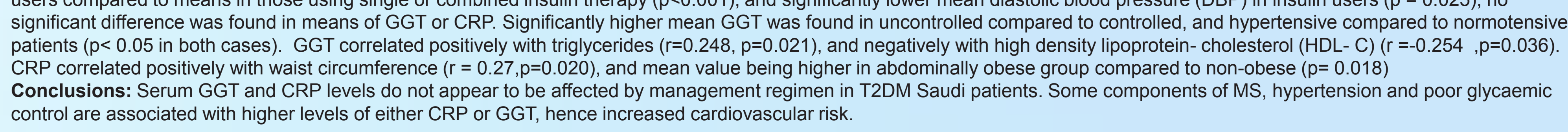

Results:

A total of 153 subjects

Table 1: Anthropometric, demographic and clinica characteristics of study group

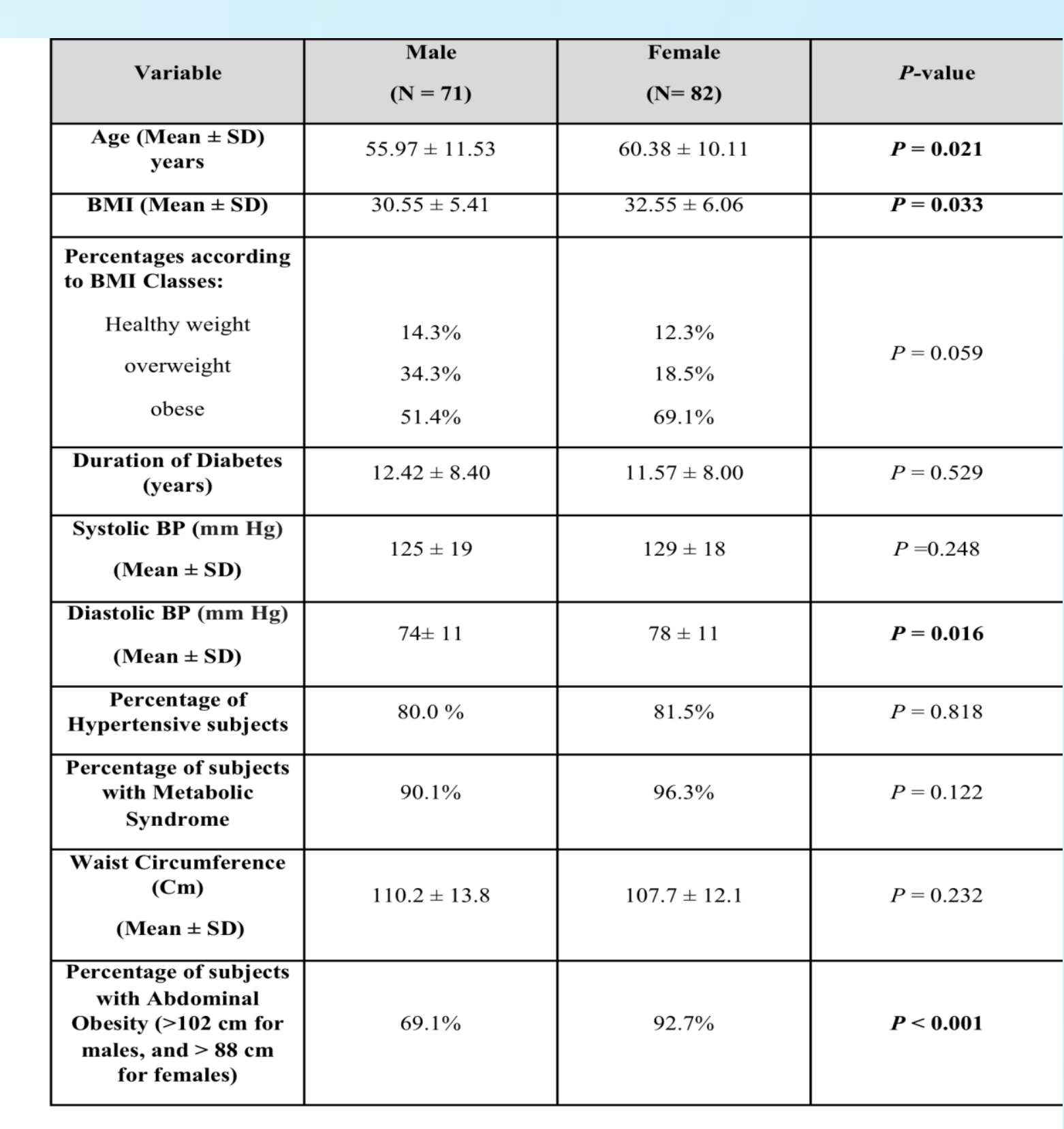

Female subjects had a significantly higher mean age, and BMI, and DBP. Females also had a significantly higher

pressure; expressed as mean $\pm \mathrm{SD}$; after

dviding subjects according to treatment modality are

Table 2: Measured biochemical variables and blo
according to treatment modality (Mean \pm SEM)

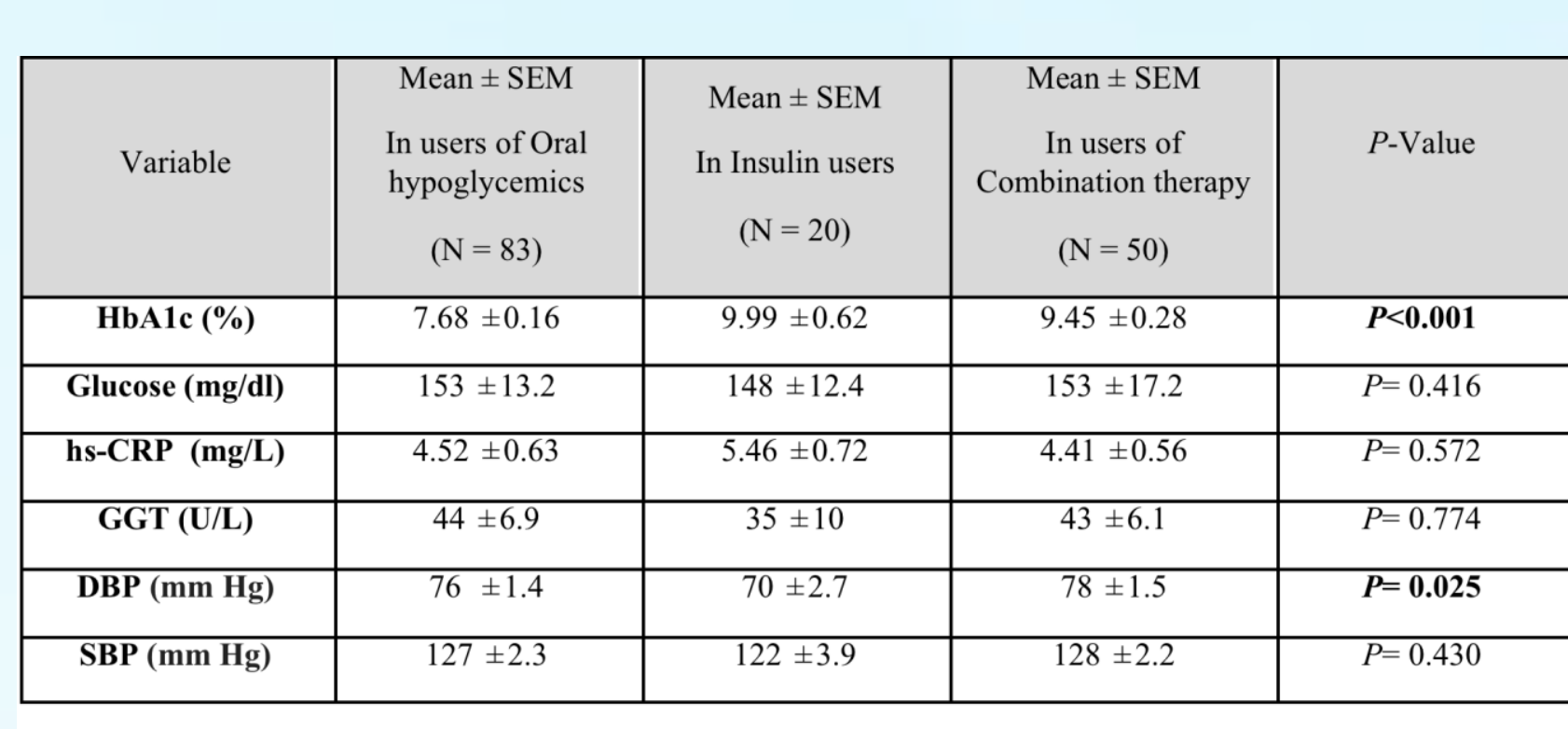

spite of significantly lower mean HbA1c in hypoglycaem drugs users compared to means in those using single or combined insulin therapy $(p<0.001)$, and significantly lower
mean diastolic blood pressure (DBP) in insulin users $(p=$ mean diastolic blood pressure (DBP) in insulin users $(p=$ Comparing mean GGT in subjects with good glycaemic
control to mean in uncontrolled subjects, the latter was foun to be significantly higher $(P=0.008)$. Significantly higher
mean $G G T$ was also found in hypertensive compared to
Figure 1: GGT level (Mean $\pm \mathrm{SD}$ ) in subjects with adequate

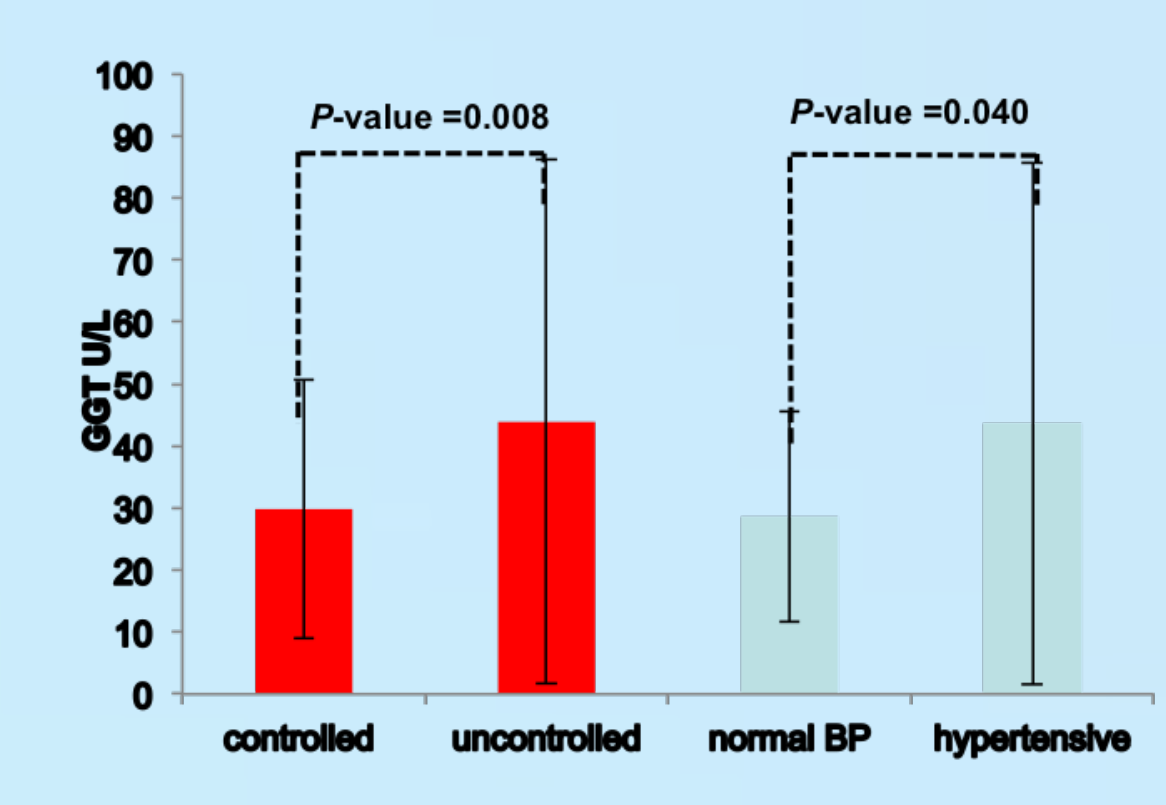

Studying the relationship between levels of GGT and hsCRP in all subjects and different components of the metabolic
syndrome, GGT was found to correlate positively with syndrome, GGT was found to correlate positively with
triglycerides, and negatively with HDL- C, but hsCRP correlated positively with waist circumference only (Table Furthermore, the mean value of hsCRP was significantly higher in abdominally obese group compared to non-obes (Figure 2)

Table 3: Correlations of hs-CRP, and GGT with different components of metabolic syndrome in all studied subjects.

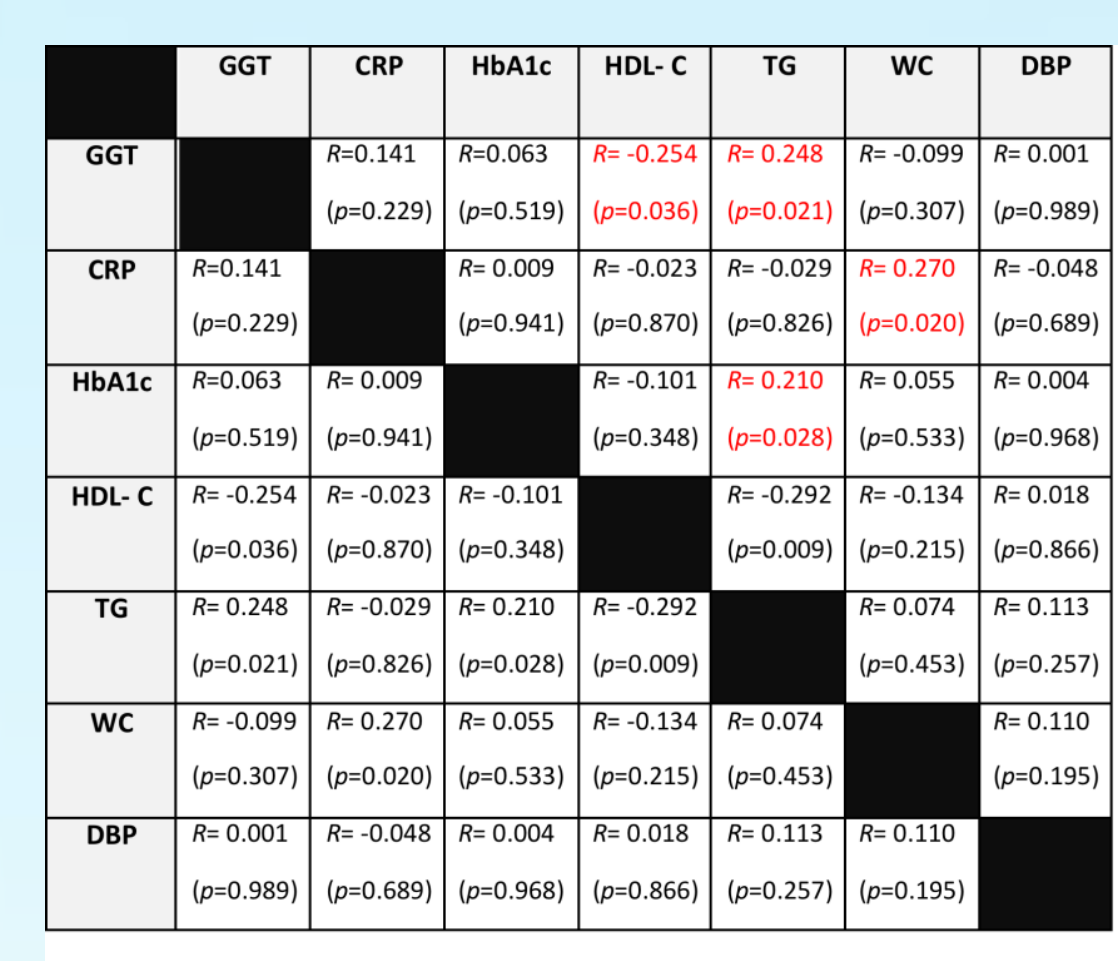

Figure 2: hs-CRP concentrations in subjects with normal and abdominal obesity (Mean \pm SD)

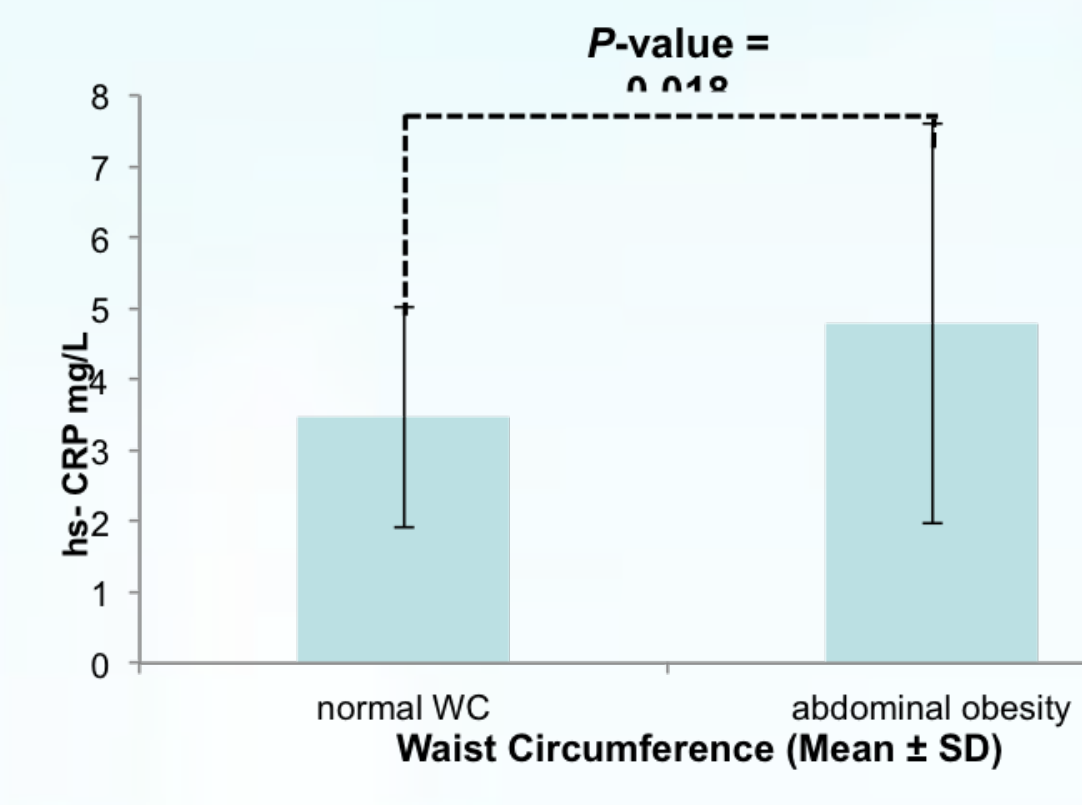
glycaemic control vs. uncontrolled subjects, and in

\section{Discussion:}

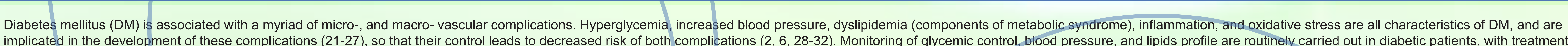

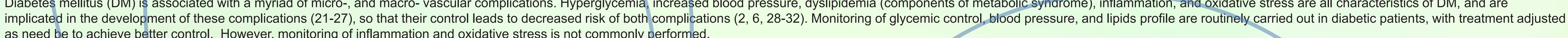

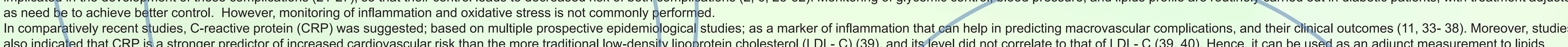

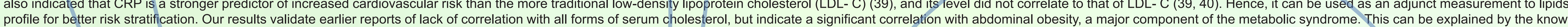

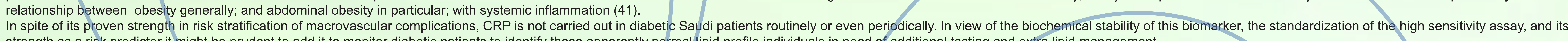

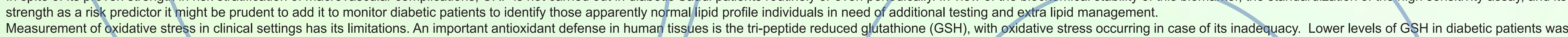

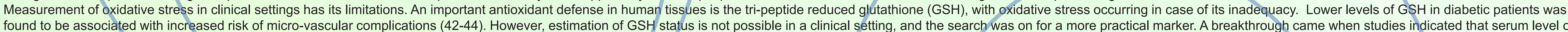

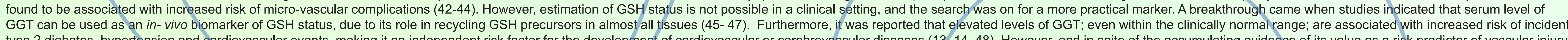

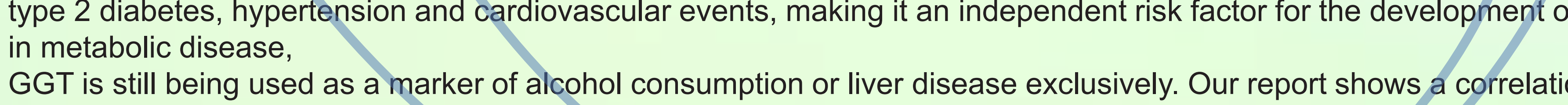
列 - conclusions:

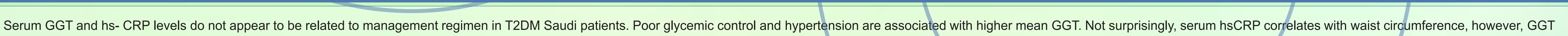

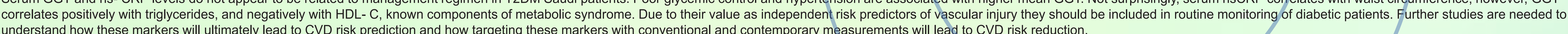

\section{Referencies:}

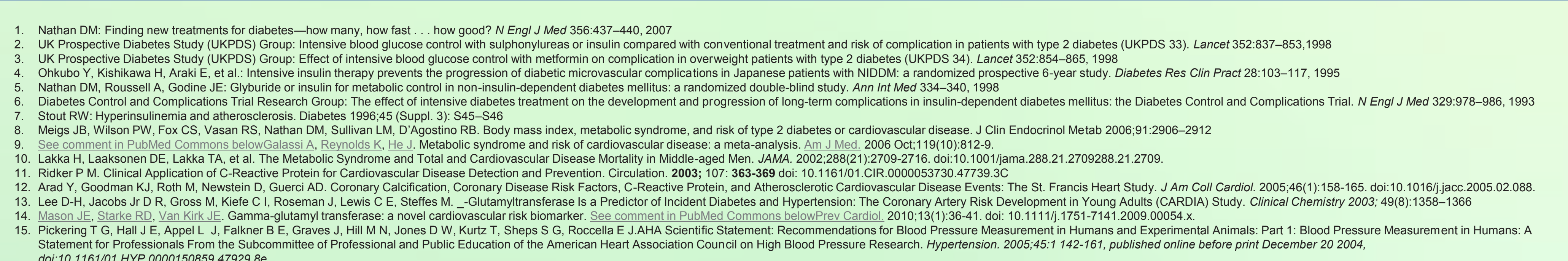

